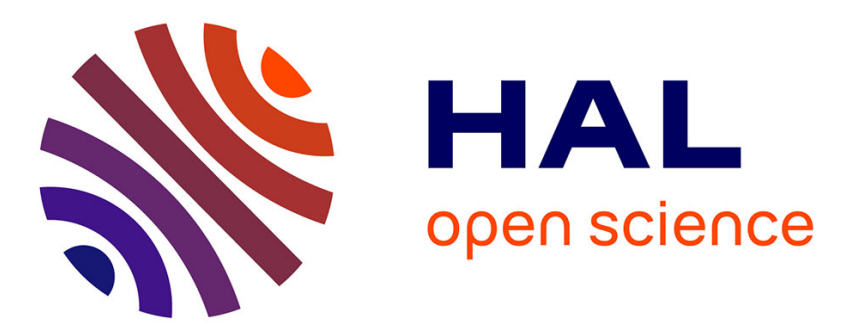

\title{
Getting a morphological tree of shapes for multivariate images: Paths, traps, and pitfalls \\ Edwin Carlinet, Thierry Géraud
}

\section{To cite this version:}

Edwin Carlinet, Thierry Géraud. Getting a morphological tree of shapes for multivariate images: Paths, traps, and pitfalls. 21st IEEE International Conference on Image Processing (ICIP), Oct 2017, Paris, France. pp.615 - 619, 10.1109/ICIP.2014.7025123 . hal-01476227

\section{HAL Id: hal-01476227 \\ https://hal.inria.fr/hal-01476227}

Submitted on 24 Feb 2017

HAL is a multi-disciplinary open access archive for the deposit and dissemination of scientific research documents, whether they are published or not. The documents may come from teaching and research institutions in France or abroad, or from public or private research centers.
L'archive ouverte pluridisciplinaire HAL, est destinée au dépôt et à la diffusion de documents scientifiques de niveau recherche, publiés ou non, émanant des établissements d'enseignement et de recherche français ou étrangers, des laboratoires publics ou privés. 


\section{GETTING A MORPHOLOGICAL TREE OF SHAPES FOR MULTIVARIATE IMAGES: PATHS, TRAPS, AND PITFALLS}

\author{
Edwin Carlinet ${ }^{1,2}$ \\ ${ }^{1}$ EPITA Research and Development Lab. (LRDE) \\ 14-16, rue Voltaire \\ FR-94276 Le Kremlin-Bicêtre, France \\ \{firstname.lastname\}@1rde.epita.fr
}

\begin{abstract}
The tree of shapes is a morphological tree that provides an high-level hierarchical representation of the image suitable for many image processing tasks. This structure has the desirable properties to be self-dual and contrast-invariant and describes the organization of the objects through level lines inclusion. Yet it is defined on gray-level while many images have multivariate data (color images, multispectral images...) where information are split across channels. In this paper, we propose some leads to extend the tree of shapes on colors with classical approaches based on total orders, more recent approaches based on graphs and also a new distance-based method. Eventually, we compare these approaches through denoising to highlight their strengths and weaknesses and show the strong potential of the new methods compared to classical ones.
\end{abstract}

Index Terms - Mathematical Morphology; Tree of Shapes; Color Image Processing; Filtering.

\section{INTRODUCTION}

Mathematical morphology (MM) offers a non-linear image processing framework which is both simple and efficient. It has been widely used in many image processing tasks as for filtering, object detection, segmentation... Behind the scene, $\mathrm{MM}$ operators rely on an ordering relation on values which must form a lattice. Those operators usually apply to binary and grayscale images, even if there are many attempts to deal with color images [1].

A particular class of MM operators, named connected operators, have been widely investigated thanks to their contourpreserving properties. Several authors $[2,3,4]$ propose to represent the inclusion of connected components in hierarchical structures: the min-tree (resp. max-tree) states the inclusion of the lower (resp. upper) level sets. Since then, many connected operators have been developed on those trees. A selfdual hierarchical representation of the image has been proposed in [5], which merges the min-tree and the max-tree into a unique structure, called the tree of shapes. Despite its large potential in many image processing and computer vision applications $[6,7,8,9]$, it is still under-exploited.

\author{
Thierry Géraud ${ }^{1,2}$ \\ ${ }^{2}$ Université Paris-Est, \\ Laboratoire d'Informatique Gaspard-Monge (LIGM) \\ A3SI, ESIEE Paris, Cité Descartes, BP 99 \\ FR-93162 Noisy-le-Grand, France
}
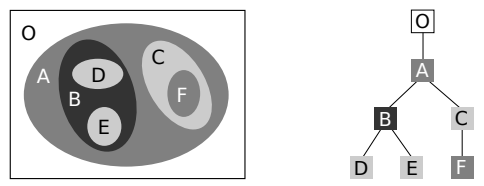

Fig. 1. An image and its tree of shapes: the components of this tree are here represented by sub-trees (so the node B corresponds to the component $B \cup D \cup E$ ).

While most MM filters rely on a partial ordering relation only, component-trees and trees of shapes need the ordering to be total. The latter requirement hinders the extension of these structures to color images. Some of the many suggested workarounds are reviewed in Section 2. Basically, they consist either in defining a total (pre)order on colors or dealing directly with the partial order on a structure which is not a tree anymore. The former approach is illustrated in Section 3 as a straightforward extension of the tree of shapes to colors. The contributions of this paper are:

- a natural extension of the algorithm [10] from Géraud et al. using an alternative definition of shapes,

- a graph approach to organize the set of shapes and deal directly with the partial order.

Both approaches are investigated in Sections 4 and 5 respectively. Section 6 eventually show a preliminary comparison of these methods through denoising. Last we conclude and give perspectives in Section 7.

\section{HIERARCHICAL STRUCTURES ON MULTIVARIATE DATA}

\subsection{Processing Colors with Trees}

Among hierarchical representations of images, component trees rely on a data (pixel values) ordering that needs to be total. When dealing with multivariate data, authors generally have two approaches. They either define a new total ordering relation on their data, or adapt their structures to deal with the natural partial order. Many authors have attempted to define orders on colors (see for example [11, 12] or Aptoula and Lefèvre [13], Velasco-Forero and Angulo [14] for a rather 
complete survey of color orders for MM processing) but few of them [15, 16, 17] have been involved in studying their influences on component trees (and even fewer on the tree of shapes [18]). While it may seem straightforward to construct a component tree (or a tree of shapes) once a total ordering relation is chosen, there are still some issues about the reconstruction process in case of preorders [15, 17]. Few people have been involved in the second approach that considers the structure implied by a partial order. However, some recent works $[19,20,21]$ have proposed to generalize the component trees to component graphs, and that idea can be transposed to the tree of shapes.

\subsection{Reminders about the Tree of Shapes}

Let $u$ be an image defined over a domain $\Omega$ (typically $\mathbb{Z}^{2}$ for 2D images), with values on a set $V$ equipped with an ordering relation. We note $\leq$ whenever the considered relation is total and $\preceq$ whenever it is partial.

The lower level set at level $\lambda \in V$ is defined by $[u \leq \lambda]=$ $\{p \in \Omega, u(p) \leq \lambda\}$; the upper level set $[u \geq \lambda]$ is defined similarly. Level sets will also be called cuts (as in [10]) in the rest of the paper. Let $\mathcal{C C}(X) \subset \mathcal{P}(\Omega)$ denote the connected components of the set $X \subset \Omega, \mathcal{L}_{\lambda}=\mathcal{C C}([u \leq \lambda])$ the lower components at level $\lambda$, and $\mathcal{L}=\cup_{\lambda \in V} \mathcal{L}_{\lambda}$ the set of lower connected components. The set $\mathcal{L}$ endowed with the inclusion relationship is a hierarchical structure named the min-tree. Its dual $\mathcal{U}$, based on upper level sets, forms the max-tree.

Let $X \subset \Omega$ and $c c(\bar{X}, \partial \Omega)$ be the connected component of $X$ 's complement which connects with the domain's border. Then, let Sed be the hole filling operator defined as $\operatorname{Sed}(X)=\Omega \backslash c c(\bar{X}, \partial \Omega)$. Then, a shape is a connected components without holes from $\mathcal{L}$ and $\mathcal{U}$. We call a lower shape of level $\lambda$, an element of $\mathcal{S}_{\lambda}^{-}=\left\{\operatorname{Sed}(X), X \in \mathcal{L}_{\lambda}\right\}$, the whole set of lower shapes is noted $\mathcal{S}^{-} . \mathcal{S}_{\lambda}^{+}$and $\mathcal{S}^{+}$are defined in the same way from $\mathcal{U}_{\lambda}$. Any two elements from the set of shapes $\mathcal{S}=\mathcal{S}^{+} \cup \mathcal{S}^{-}$being either disjoint or nested, $(\mathcal{S}, \subseteq)$ also forms a tree called the tree of shapes.

\subsection{Grain Filters and Reconstruction}

Given a total order $(V, \leq)$, an image can be reconstructed from the set of shapes by:

$$
\begin{aligned}
\tilde{u}(x) & =\max \left\{\lambda \mid x \in A \in \mathcal{S}_{\lambda}^{-}, x \notin B \in \mathcal{S}_{\lambda^{\prime}>\lambda}^{+}, B \subset A\right\} \\
& =\min \left\{\lambda \mid x \in A \in \mathcal{S}_{\lambda}^{+}, x \notin B \in \mathcal{S}_{\lambda^{\prime}<\lambda}^{-}, B \subset A\right\}
\end{aligned}
$$

The grain filter consists in removing shapes having a size below a given threshold and reconstructing from a subset of $\mathcal{S}$. Algorithmically speaking, filtering means pruning the tree of shapes and merging the deleted nodes with the first parent node verifying the criterion. Reconstructing consists in assigning to each pixel, the level of the node it belongs to.

\section{TOTAL (PRE-)ORDERS APPROACH}

A classification of ordering relations can rely on their algebraic properties (totality, anti-symmetry...) or with respect to the way these relations are built. Barnett [22] proposed to classify them into four groups: marginal (M-ordering), conditional (C-ordering), partial (P-ordering) and reduced (R-ordering). The former is a component-wise ordering that deals separately with each channel, i.e., a partial order, and it will be discussed in Section 5. The three others provide total (pre)orders and are discussed below.

With C-ordering, vectors are ordered by mean of one, several, or all of their marginal components. The most wellknown C-ordering is the lexicographical ordering, that is a total order. If only several components participate in the comparison, it yields a total-preorder. For example let $v, w \in \mathbb{R}^{n}$, the lexicographical ordering $\leq_{L}$ using only the two first components $\left(v \leq_{L} w\right.$ iff $\left.v_{1}<w_{1} \vee v_{1}=w_{1} \wedge v_{2} \leq w_{2}\right)$ is a total-preorder. Colors $(1,1,2)$ and $(1,1,3)$ are considered as equivalent by the above relation. The main pitfall of $\mathrm{C}$ orderings lies in the importance given to the first components. Considering the RGB space for example, it implies that the red component is more relevant than the others. Workarounds like the sub-quantization of first components (also known as $\alpha$-lexicographical ordering [23, 24]) enables to lower the importance given to the first dimension.

With R-ordering, vectors are reduced to scalar values using a mapping $h: \mathbb{R}^{n} \rightarrow \mathbb{R}$ s.t $v<w \Leftrightarrow h(v)<h(w)$. If $h$ is injective then each index is mapped to a unique color and the relation is a total order, otherwise it is a total preorder. Typical examples of R-ordering are distance-based ordering. It consists in choosing a reference vector (or reference set of vectors) $v_{\text {ref }}$ and the order relation is built upon a distance to $v_{r e f}\left(v \leq_{R} w\right.$ iff $\left.d\left(v, v_{r e f}\right) \leq d\left(w, v_{r e f}\right)\right)$. The main drawback of distance-based orders lies in the choice of reference vectors. The method in Section 4 can be seen as a local Rordering were the reference vector is the parent shape level.

Image Restitution. While some applications (e.g., object detection, segmentation) are not concerned with the restitution of the image, filtering requires reconstructing from the pruned tree. If the order used to construct the tree is a not anti-symmetric (preorder), then a single node is associated with several levels and reconstruction issues arise. In [15], two strategies are proposed: $P_{\text {mean }}$ and $P_{\text {median }}$. During restitution, the former assigns the mean value of the node's pixels whereas the latter assigns the median value (based on the total lexicographical order). Tushabe and Wilkinson [17] extend those ideas with: (1) reconstructing the removed shapes with the mean color of the parent shape and leave others pixels intact (Mean Parent (MP)), (2) reconstructing the removed shapes with the closest color in the parent shape (Nearest Color $(N C)$ ) and (3) computing the geodesic influence of the parent shape pixels in the removed shape and assigning the closest pixel color (Nearest Neighbor $(N N)$ ). The last strategy is not a connected filter, thus it is not considered in the comparison in Section 6.

\section{A DISTANCE-BASED APPROACH}

While the initial definition of the tree of shapes $[25,26]$ relies on the upper and lower level sets only, it encodes two pieces of information: the level sets and the level lines. In Morse theory, level lines are either defined by iso-contours 
$[u=\lambda]$ or in terms of contours of lower or upper level sets. In discrete topology, the second definition is more convenient because it does not require $u$ to be continuous. Nevertheless, a recent tree of shapes computation algorithm [10] uses a continuous interpretation scheme of the image using an intervalvalued map on a Khalimsky grid [27] where isolines are welldefined. The algorithm works as follows: it starts a propagation from the border of the image and floods every level set in a continuous way, i.e., if $\lambda$ is the level currently flooded, the next flooded level is either $\lambda+1$ or $\lambda-1$. Once the image has been totally flooded, a union-find step processes the pixels in the reverse order of the propagation and builds the tree of shapes. The algorithm thus provides a recursive definition of shapes in gray levels: given a shape $s_{\lambda}$ at level $\lambda$, one can generate a set $S^{\prime}$ of $s_{\lambda}$ sub-shapes with:

$$
\begin{aligned}
& S^{\prime}=\left\{s_{\lambda^{\prime}}=\operatorname{Sed}(X), X \in \mathcal{C C}\left(s_{\lambda} \backslash[u=\lambda]\right)\right\} \\
& \text { with } \lambda^{\prime}=\lambda+1 \text { or } \lambda^{\prime}=\lambda-1 .
\end{aligned}
$$

Definition given by Equation (2) and the algorithm ensure that shapes will form a tree. However, it requires to choose a next flooding level $\lambda^{\prime}$ in order to continue the propagation in a hole. In grayscale, we choose either $\lambda+1$ or $\lambda-1$ which is the closest upper or lower level available in the hole's border. To mimic this continuous behavior, we propose to choose also the closest level in terms of a distance which is equivalent to impose locally a total (pre)order based on a distance to a reference color. Equation (2) becomes:

$$
\begin{aligned}
S^{\prime}=\left\{s_{\lambda^{\prime}}\right. & \left.=\operatorname{Sed}(X), X \in \mathcal{C C}\left(s_{\lambda} \backslash[u=\lambda]\right)\right\} \\
\text { with } \lambda^{\prime} & =\min _{v \in u(\partial \operatorname{Sed}(X))}\|\lambda-v\|
\end{aligned}
$$

Note that Equation (3) is equivalent to the original definition in gray levels. Also, since $\lambda^{\prime}$ may not be unique, one can combine the distance with a lexicographical cascade to avoid the pitfall of multiple minima.

\section{A GRAPH APPROACH}

Since a natural total order is not obvious, some approaches try to deal with the natural partial order that does exist. A set of shapes $\mathcal{S}$ is endowed with the inclusion relationship, the Haas diagram of $(\mathcal{S}, \subseteq)$ is a directed acyclic graph (DAG) which is our processing structure. We consider two sets for $\mathcal{S}$ :

1) $\dot{\mathcal{S}}=\left\{\operatorname{Sed}(X), X \in \mathcal{L}_{\preceq} \cup \mathcal{U}_{\succeq}\right\}$

2) $\ddot{\mathcal{S}}=\left\{\operatorname{Sed}(X), X \in \bigcup_{i} \mathcal{C C}\left(\left[u_{i} \leq \lambda_{i}\right]\right) \cup \mathcal{C C}\left(\left[u_{i} \geq \lambda_{i}\right]\right)\right\}$

In other words, $\dot{\mathcal{S}}$ is the set of all shapes extracted from every cuts $[u \preceq \lambda]$ and $[u \succeq \lambda]$, while $\mathcal{\mathcal { S }}$ is the set of marginal shapes. In Figure 2, where $\dot{\mathcal{G}}$ denotes the shape-graph associated with the cover relation of $(\dot{\mathcal{S}}, \subseteq)$ and $\ddot{\mathcal{G}}$ the one of $(\ddot{\mathcal{S}}, \subseteq)$, we highlight the difference between the two sets of shapes and shows that $\dot{\mathcal{G}}$ is finer that $\ddot{\mathcal{G}}$. Actually, any lower marginal cut can be obtained from $\left[u \preceq\left(\top, \ldots, \top, \lambda_{i}, \top, \ldots, \top\right)\right]$ (and upper marginal cuts as well), thus $\ddot{\mathcal{S}} \subset \dot{\mathcal{S}}$. However, $\ddot{\mathcal{S}}$ and $\ddot{\mathcal{G}}$ have an interest from a computational point of view because, contrary to $\dot{\mathcal{G}}, \ddot{\mathcal{G}}$ can be computed efficiently by merging the marginal trees of shapes built on individual channels.

Filtering and Reconstruction. One can reconstruct $u$ from

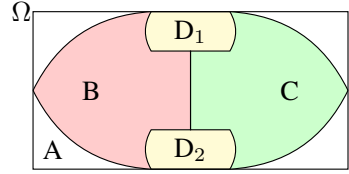

Original image $u$

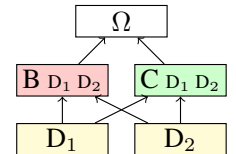

Shape-graph $\dot{\mathcal{G}}$

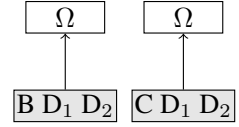

$\mathcal{T}_{1}$ and $\mathcal{T}_{2}$

Trees of Shapes

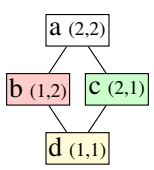

$(V, \preceq)$



Shape-graph $\ddot{\mathcal{G}}$
Fig. 2. Graph-shapes $\dot{\mathcal{G}}$ and $\ddot{\mathcal{G}}$.


(a) Original image $u$ and its shape-graph $\ddot{\mathcal{G}}$. Light nodes with a dashed border represent cuts of colors missing in $u$. Nodes with a red border are the shapes to be removed.

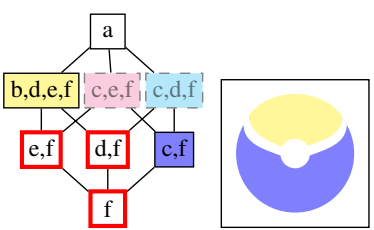

(b) Filtering with the LCA strategy

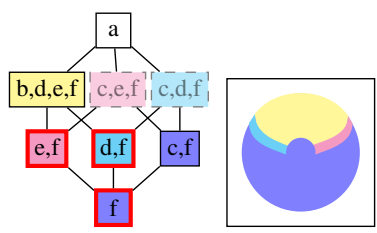

(c) A possible filtering with the NC strategy
Fig. 3. Filtering $\dot{\mathcal{G}}$.

$\dot{\mathcal{G}}$ using the same principle of Equation (1) where the $\max$ and min are respectively replaced by the infimum and the supremum. Algorithmically speaking, a node of the DAG $\dot{\mathcal{G}}$ is filtered with the value of the least common ancestor (LCA) of its parents (LCA strategy). If the LCA is both a lower and an upper shape with different level, the mean of the levels is chosen as the restitution value. This restitution rule tends to create color artifacts since the LCA may be distant in the DAG hierarchy. Thus, we propose another strategy derived from [17]. A removed node is filtered with the color of the closest (in terms of the color distance) parent node ( $N C$ strategy). In Figure 3a, the least common ancestor of the shapes to remove is the root, thus the LCA strategy reconstructs the shapes with value $(1,1,1)$. On the contrary, with the NC strategy, the $e, f$ shape can be reconstructed with value $(1,1,0)$ or $(1,0,1)$ depending on the chosen distance. Those values are closer to the original one $(1,0,0)$. Reconstructing $u$ from $\ddot{\mathcal{G}}$ is straightforward and results in a component-wise restitution, i.e., $\tilde{u}(x)=\left(\tilde{u}_{1}(x), \ldots, \tilde{u}_{n}(x)\right)$ where $\tilde{u}_{i}(x)$ is given by Equation (1) based on shapes $\ddot{\mathcal{S}}_{i, \lambda}^{+}$and $\ddot{\mathcal{S}}_{i, \lambda}^{-}$. 


\begin{tabular}{|c|c|c|c|c|}
\hline & $\begin{array}{r}\text { Best Grain } \\
\text { Size }\end{array}$ & $\begin{array}{r}\text { Min } \\
\text { PSNR }\end{array}$ & $\begin{array}{r}\text { Max } \\
\text { PSNR }\end{array}$ & $\begin{array}{l}\text { Avg. } \\
\text { PSNR }\end{array}$ \\
\hline Shape-Graph $\dot{\mathcal{G}}$ & \multicolumn{4}{|c|}{ N/A ( $\dot{\mathcal{G}}$ computation is a non-tractable problem) } \\
\hline Preorder $C_{\mathrm{La}^{*} \mathrm{~b} *}(\mathrm{NC})$ & 2 & 36.21 & 39.60 & 37.43 \\
\hline Preorder $L_{\mathrm{La}^{*} \mathrm{~b} *}(\mathrm{NC})$ & 5 & 36.32 & 38.43 & 37.43 \\
\hline Preorder $L_{\mathrm{HLS}}(\mathrm{NC})$ & 7 & 36.34 & 39.36 & 37.57 \\
\hline Preorder Brightness (NC) & 8.5 & 36.39 & 39.60 & 37.67 \\
\hline Order Lex & 100 & 36.42 & 47.78 & 38.10 \\
\hline Distance-Based & 15 & 37.02 & 41.14 & 38.76 \\
\hline Preorder $C_{\mathrm{La}^{*} \mathrm{~b} *}(\mathrm{MP})$ & 300 & 36.31 & 41.37 & 38.92 \\
\hline Preorder $C_{\mathrm{La}^{*} \mathrm{~b} *}\left(P_{\text {mean }}\right)$ & 200 & 36.20 & 41.45 & 39.03 \\
\hline Preorder $L_{\mathrm{La}^{*} \mathrm{~b} *}(\mathrm{MP})$ & 300 & 36.77 & 41.38 & 39.34 \\
\hline Preorder $L_{\mathrm{HLS}}(\mathrm{MP})$ & 300 & 36.65 & 41.49 & 39.59 \\
\hline Preorder $L_{\mathrm{La}^{*} \mathrm{~b} *}\left(P_{\text {mean }}\right)$ & 200 & 37.00 & 42.22 & 39.72 \\
\hline Preorder Brightness (MP) & 300 & 36.60 & 42.69 & 39.94 \\
\hline Preorder $L_{\mathrm{HLS}}\left(P_{\text {mean }}\right)$ & 200 & 37.03 & 41.84 & 39.96 \\
\hline Preorder Brightness $\left(P_{\text {mean }}\right)$ & 200 & 36.41 & 43.19 & 40.40 \\
\hline Shape-Graph $\ddot{\mathcal{G}}$ (marginal) & 50 & 37.59 & 50.21 & 41.66 \\
\hline
\end{tabular}

Table 1. Min, max, and average PSNR scores of the methods

\section{EXPERIMENTS}

We compare the different approaches for denoising. Even if this application does not take advantage of high-level abstraction provided by the Tree/Graph of Shapes, it is still a good assessment of the meaningfulness and the coherence of the representation. The dataset is composed by 65 color images with a strong chromatic component used for image compression and color constancy test [28,29]. A Gaussian noise $(\mu=$ $0, \sigma=30)$ is first applied independently on each channel and we then compare the three approaches: total (pre)orderbased, distance-based and graph-based. For the first category, we evaluate the $R G B$ lexicographical order (namely Lex) and preorders based on lightness (in La*b* and HLS spaces), brightness (as the average of the three RGB components) and chrominance (in $\mathrm{La}^{*} \mathrm{~b} *$ space, $C=\sqrt{a^{2}+b^{2}}$ ). They are

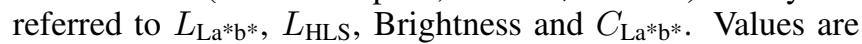
quantized to 256 levels and each method was tested with the $M P, N C$ and $P_{\text {mean }}$ strategies. The second and third classes of methods are those described in Sections 4 and 5. We use the Peak Signal to Noise Ratio (PSNR) to assess the denoising quality. We apply grain filters of size 2 to 500, and we retain the highest PSNR score for each pair (method, image) ${ }^{1}$.

Table 1 shows the min, max and average PSNR score for each method, as well as the average grain size $\lambda$ for which the maximum score has been obtained. The shape-graph approach outperforms the others, then come preorders based on $P_{\text {mean }}$ and $M P$ strategies and then, the distance-based approach. The Nearest Color strategy performs the worst in our benchmark. It is not surprising that the best methods are those that tend to blur the image by averaging color when reconstructing $(M P$, and $P_{\text {mean }}$ ) or by reconstructing marginally (Shape-Graph $\ddot{\mathcal{G}}$ ). Even if they create false colors (colors not in the original image), the new colors may (or may not) make sense in the original image. Indeed, in Figure 4e, the false colors generated by marginal reconstruction are close to the original ones. In Figures $4 \mathrm{~d}$ and $4 \mathrm{f}$, false colors create visible artifacts (in the back-

\footnotetext{
${ }^{1}$ An in-depth description of the evaluation and complete results are available on http://Irde.epita.fr/wiki/Publications/ carlinet.14.icip
}

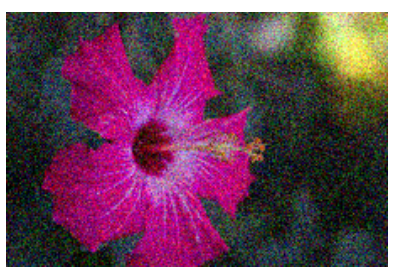

(a) Original image with gaussian noise corruption. PSNR=36.46

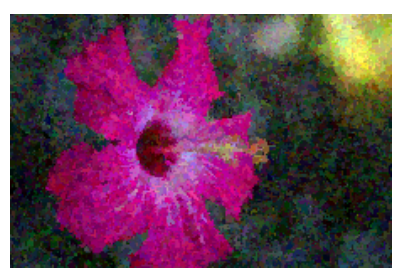

(c) Distance-based $\lambda=9$, PSNR $=37.88$

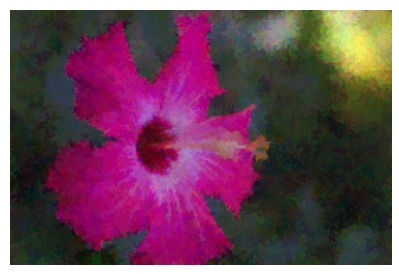

(e) Shape-Graph $\ddot{\mathcal{G}}$ (marginal). $\lambda=30$, PSNR $=39.98$

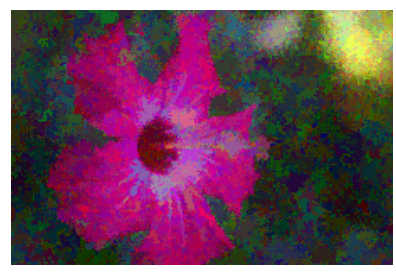

(b) Preorder Brightness (NC). $\lambda=100$, PSNR $=37.24$

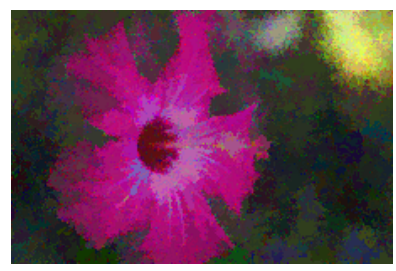

(d) Preorder Brightness (MP). $\lambda=100$, PSNR $=37.98$

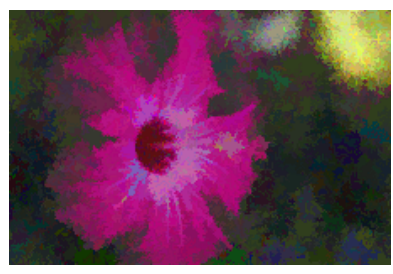

(f) Preorder Brightness $\left(P_{\text {mean }}\right)$. $\lambda=100$, PSNR $=38.23$
Fig. 4. Top 5 methods performing the best denoising on the flower image.

ground and the flower) due to averaging pixels with a similar brightness but a different chrominance. Figure $4 \mathrm{~b}$ has even more visible artifacts for different reasons: no false colors are created (since shapes are assigned with closest colors from the image) but it may reconstruct with a noise pixel value. Also, (pre)order based methods require a high filtering grain size to perform a good reconstruction (see the first column of Table 1) meaning that they cannot retain details in presence of noise. On the contrary, the distance-based approach preserves the details while preventing most color artifacts (Figure 4c). Thus it is well adapted for fine objects retrieval.

\section{CONCLUSION}

We have presented three classes of methods to extend the tree of shapes to multivariate images that have been evaluated through a denoising contest. We have shown that classical approaches consisting in equipping the data with a total order or a total preorder perform well but highly depend on the restitution rules and suffer from color artifacts. We have also presented two new approaches: the first is an algorithmic redefinition of shapes based on distances, and the second is the component-graph extended to the tree of shapes. They both produced promising results in our evaluation that highlights their strong potential for more complex tasks in computer vision. In an incoming work, we will further investigate the capacities of theses methods for object retrieval and simplification of images where chrominance matters by adapting tree of shapes processing algorithms to shape-graphs. 


\section{References}

[1] J. Angulo and J. Chanussot, "Color and multivariate images," in Mathematical Morphology-From Theory to Applications, L. Najman and H. Talbot, Eds. ISTE \& Wiley, 2010, ch. 11, pp. 291-321.

[2] E. Breen and R. Jones, "Attribute openings, thinnings and granulometries," Computer Vision and Image Understanding, vol. 64, no. 3, pp. 377-389, 1996.

[3] P. Salembier, A. Oliveras, and L. Garrido, "Antiextensive connected operators for image and sequence processing," IEEE Transactions on Image Processing, vol. 7, no. 4, pp. 555-570, 1998.

[4] R. Jones, "Connected filtering and segmentation using component trees," Computer Vision and Image Understanding, vol. 75, no. 3, pp. 215-228, 1999.

[5] P. Monasse and F. Guichard, "Fast computation of a contrast-invariant image representation," IEEE Trans. Image Process., vol. 9, no. 5, pp. 860-872, 2000.

[6] C. Ballester, V. Caselles, L. Igual, and L. Garrido, "Level lines selection with variational models for segmentation and encoding," Journal of Mathematic Imaging and Vision, vol. 27, pp. 5-27, 2007.

[7] J. Cardelino, G. Randall, M. Bertalmio, and V. Caselles, "Region based segmentation using the tree of shapes," in Proc. of IEEE International Conference on Image Processing, 2006, pp. 2421-2424.

[8] Y. Xu, T. Géraud, and L. Najman, "Context-based energy estimator: Application to object segmentation on the tree of shapes," in Proc. of IEEE International Conference on Image Processing, Orlando, Florida, USA, 2012, pp. 1577-1580.

[9] Y. Xu, T. Géraud, and L. Najman, "Salient level lines selection using the Mumford-Shah functional," in Proc. of IEEE International Conference on Image Processing, 2013, pp. 1227-1231.

[10] T. Géraud, E. Carlinet, S. Crozet, and L. Najman, "A quasi-linear algorithm to compute the tree of shapes of $n$-D images." in Proc. of Intl. Symp. on Mathematical Morphology (ISMM), ser. LNCS, vol. 7883. Springer, 2013, pp. 98-110.

[11] O. Lezoray, C. Charrier, A. Elmoataz et al., "Rank transformation and manifold learning for multivariate mathematical morphology," in Proc. of European Signal Processing Conference, vol. 1, 2009, pp. 35-39.

[12] S. Velasco-Forero and J. Angulo, "Supervised ordering in $\mathcal{R}_{p}$ : application to morphological processing of hyperspectral images." IEEE Transactions on Image Processing, vol. 20, no. 11, p. 3301, 2011.

[13] E. Aptoula and S. Lefèvre, "A comparative study on multivariate mathematical morphology," Pattern Recognition, vol. 40, no. 11, pp. 2914-2929, 2007.

[14] S. Velasco-Forero and J. Angulo, "Vector ordering and multispectral morphological image processing," in $A d$ - vances in Low-Level Color Image Processing, ser. Lecture Notes in Computational Vision and Biomechanics. Springer, 2014, vol. 11, pp. 223-239.

[15] B. Naegel and N. Passat, "Component-trees and multivalue images: A comparative study," in Proc. of Intl. Symp. on Mathematical Morphology (ISMM), ser. LNCS, vol. 5720. Springer, 2009, pp. 261-271.

[16] B. Perret, S. Lefèvre, C. Collet, and E. Slezak, "Connected component trees for multivariate image processing and applications in astronomy," in Proc. of International Conference on Pattern Recognition, Aug. 2010, pp. 4089-4092.

[17] F. Tushabe and M. H. F. Wilkinson, "Color processing using max-trees: A comparison on image compression," in Proc. of International Conference on Systems and Informatics (ICSAI). IEEE, 2012, pp. 1374-1380.

[18] V. Caselles and P. Monasse, Geometric description of images as topographic maps, ser. Lecture Notes in Mathematics. Springer-Verlag, 2009, vol. 1984.

[19] N. Passat and B. Naegel, "An extension of componenttrees to partial orders," in Proc. of IEEE International Conference on Image Processing, 2009, pp. 3981-3984.

[20] N. Passat and B. Naegel, "Component-trees and multivalued images: Structural properties," Journal of Mathematical Imaging and Vision, pp. 1-14, 2013.

[21] B. Naegel and N. Passat, "Towards connected filtering based on component-graphs," in Proc. of Intl. Symp. on Mathematical Morphology (ISMM), ser. LNCS, vol. 7883. Springer, 2013, pp. 353-364.

[22] V. Barnett, "The ordering of multivariate data," Journal of the Royal Statistical Society. Series A (General), vol. 139, no. 3, pp. 318-355, 1976.

[23] J. Angulo, "Unified morphological color processing framework in a Lum/Sat/Hue representation," in Proc. of Intl. Symp. on Mathematical Morphology (ISMM), ser. Computational Imaging and Vision, vol. 30. Springer, 2005, pp. 387-396.

[24] J. Serra, "Représentations de la couleur en coordonnées polaires adaptées au traitement d'images," 2005.

[25] V. Caselles, B. Coll, and J. Morel, "Topographic maps and local contrast changes in natural images," Intl. J. of Computer Vision, vol. 33, no. 1, pp. 5-27, 1999.

[26] V. Caselles and P. Monasse, "Grain filters," Journal of Mathematic Imaging and Vision, vol. 17, no. 3, pp. 249270, Nov. 2002.

[27] L. Najman and T. Géraud, "Discrete set-valued continuity and interpolation," in Proc. of Intl. Symp. on Mathematical Morphology (ISMM), ser. LNCS, vol. 7883. Springer, 2013, pp. 37-48.

[28] K. Barnard, L. Martin, B. Funt, and A. Coath, "A data set for color research," Color Research \& Application, vol. 27, no. 3, pp. 147-151, 2002.

[29] S. Garg, "Image compression benchmark," 2008. Available: http://www.imagecompression.info/ 